\title{
Fatores associados ao estresse da equipe de enfermagem de unidade de clínica cirúrgica
}

\section{Factors associated with stress in the nursing team of a surgical clinic unit}

\author{
Oclaris Lopes Munhoz, Rafaela Andolhe, Tânia Solange Bosi de Souza Magnago, \\ Tatiele Soares Arrial, Laura Prestes Moreira
}

Como citar este artigo: MUNHOZ, OCLARIS L.; ANDOLHE, RAFAELA; MAGNACO, TÂNIA S. B. S.; ARRIAL, TATIELE S.; MOREIRA, LAURA P.

Fatores associados ao estresse da equipe de enfermagem de unidade de clínica cirúrgica. Revista Saúde (Sta. Maria). 2020; 46 (2).

\section{Autor correspondente:}

Nome: Oclaris Lopes Munhoz

E-mail: oclaris_munhoz@hotmail.com

Telefone: (55) 99679-90 13

Formação Profissional: Enfermeiro.

Mestre em Enfermagem pela

Universidade Federal de Santa

Maria (UFSM) que fica na cidade de Santa Maria, RS, Brasil.

Filiação Institucional: Universidade Federal de Santa Maria

Endereço para correspondência:

Avenida Roraima nः: 1000

Bairro: Camobi

Cidade: Santa Maria

Estado: Rio Grande do Sul

CEP: 97 105-900

Data de Submissão:

$09 / 12 / 2019$

Data de aceite:

18/05/2020

Conflito de Interesse: Não há conflito de interesse

\section{(cc) $\mathrm{BY}-\mathrm{NC}-\mathrm{ND}$}

\section{RESUMO}

Objetivo: analisar a associação entre as características biopsicossociais e do trabalho e 0 nível de estresse de uma equipe de enfermagem de Unidade de Clínica Cirúrgica. Metodologia: estudo transversal, desenvolvido com 52 profissionais da equipe de enfermagem de uma Unidade de Clínica Cirúrgica (UCC). Para a coleta de dados utilizou-se um questionário contendo características biopsicossociais e do trabalho e a Escala de Estresse no Trabalho (EET). Na análise empregou-se estatística descritiva e inferencial. Respeitou-se os preceitos éticos que envolvem pesquisas com seres humanos. Resultados: quanto ao nível de estresse, dos 52 participantes, 50\% (26) apresentaram médio nivel de estresse, 25\% (13) estavam com baixo nível e 25\% (13) apresentaram alto nível de estresse. Não se constatou associação estatisticamente significativa entre nivel de estresse medido e as variáveis biopsicossociais e do trabalho dos profissionais de enfermagem. Conclusão: a equipe de enfermagem apresentou nível médio de estresse. Embora não se tenham constatado associações significativas entre as características biopsicossociais e do trabalho com o estresse, a UCC caracterizase por ser um cenário onde o estresse é presente.

PALAVRAS-CHAVE: Estresse fisiológico; Equipe de enfermagem; Assistência perioperatória.

\section{ABSTRACT}

Objective: to analyze the association between biopsychosocial and work characteristics and the stress level of a nursing team in the Surgical Clinics Unit. Methodology: Cross-sectional study, developed with 52 professionals from the nursing team of a Clinical Surgical Unit (UCC). For data collection, a questionnaire containing biopsychosocial and work characteristics and the Work Stress Scale (EET) was used. Descriptive and inferential statistics were used in the analysis. The ethical precepts involving research with human beings were respected. Results: as for the level of stress, of the 52 participants, 50\% (26) had medium level of stress, 25\% (13) had a low level and 25\% (13) had a high level of stress. There was no statistically significant association between the level of stress measured and the biopsychosocial and work variables of nursing professionals. Conclusion: the nursing team had an average level of stress. Although there were no significant associations between biopsychosocial characteristics and work with stress, UCC is characterized by being a scenario where stress is present.

KEYWORDS: Physiological stress; Nursing team; Perioperative care. 


\section{INTRODUÇÃO}

O estresse é considerado um dos fatores que mais atinge os profissionais de enfermagem e, consequentemente, além de ser um agravo à saúde do trabalhador, contribui para o acontecimento dos eventos adversos e incidentes (EA/I) relacionados à assistência em saúde ${ }^{1}$. Considerando a magnitude do estresse, autores vêm o definindo como sendo um elemento da modernidade ${ }^{1-3}$.

Em se tratando de conceitos, Hans Seyle define o estresse como sendo a Síndrome de Adaptação Geral (SGA) que está relacionada às respostas fisiológicas do organismo humano diante de um estressor ${ }^{1}$. Os estudos sobre 0 estresse seguem três linhas, são elas: na primeira, a resposta biológica (aspectos do sistema nervoso central, endócrinos, imunitários e do comportamento em geral); na segunda, os acontecimentos que desencadeiam o estresse; e, por último, as relações em que as circunstâncias estressantes se estabelecem com indivíduo e meio ambiente².

A equipe de enfermagem, principalmente em nível hospitalar, está mais exposta aos estressores ${ }^{3}$. Considerando essa questão, estudo realizado no Hospital Geral Universitário de Murcia, em unidades de internação, apresentou como resultado para estressores da equipe de enfermagem a sobrecarga de trabalho, as interrupções frequentes durante a execução de suas tarefas, a falta de tempo para alguns pacientes que necessitam, as consequências de erros para os pacientes e a sensação de que, ao terminar o trabalho, permanecem tarefas pendentes ${ }^{4}$. Somado a isso, o estresse afeta tanto a saúde do profissional, quanto suas relações sociais importantes para seu ambiente de trabalho; tais condições também não contribuem para a segurança do paciente, pois o foco pode ser desviado ${ }^{5,6}$.

Estudo desenvolvido com os profissionais de enfermagem atuantes em Unidades de Terapia Intensiva (UTI) cirúrgicas, clínicas e de especialidade constatou que $74,47 \%$ dos participantes encontravam-se com médio nível de estresse, $13,29 \%$ com baixo nível e outros $12,24 \%$ com alto nível de estresse ${ }^{6}$. Em outra pesquisa com a equipe de enfermagem, verificou-se que $56,7 \%$ dos profissionais apresentaram estresse, sendo 6,7\% em fase de quase-exaustão ${ }^{7}$.

Com isso, evidencia-se a importância das instituições de saúde em discutir e ampliar esta temática com as equipes de saúde, com vistas a reduzir o estresse do profissional, favorecendo a saúde do trabalhador e, consequentemente, a segurança do paciente que dele necessita. Nessa perspectiva, considera-se, ainda, a pouca visibilidade dos ambientes cirúrgicos acerca dos fatores associados ao estresse dos profissionais de saúde.

Frente ao exposto, questiona-se: quais são os fatores associados ao estresse da equipe de enfermagem da unidade de clínica cirúrgica de um hospital-escola do interior do Rio Grande do Sul, Brasil? Este estudo objetivou analisar a associação entre as características biopsicossociais e do trabalho e o nível de estresse de uma equipe de enfermagem de Unidade de Clínica Cirúrgica (UCC). Assim, espera-se encontrar evidências científicas acerca das condições que estão relacionadas ao estresse dos profissionais com vistas a fornecer subsídios para o planejamento e as melhorias do trabalho da equipe de enfermagem que atua no cenário cirúrgico, favorecendo a interface saúde do trabalhador e segurança do paciente. 


\section{MÉTODO}

Estudo transversal, desenvolvido com a equipe de enfermagem de uma Unidade de Cirurgia Geral de um hospitalescola da região central do Rio Grande do Sul, Brasil. Este hospital é referência para a região centro-oeste do Estado, e a unidade estudada atende pacientes em situações de pré-operatório e pós-operatório. Dentre as especialidades que são assistidas na unidade, estão: traumatologia, ortopedia, urologia, cabeça e pescoço, plástica, gastrenterologia, proctologia e cirurgia geral.

Foram incluídos todos os profissionais da equipe de enfermagem do campo de estudo que estiveram atuando no período de coleta de dados. Foram excluídos os que estavam afastados do trabalho por qualquer motivo. Constituiuse de uma população por conveniência e não probabilística, na qual, de um total de 67 profissionais, participaram da pesquisa 52 profissionais de enfermagem, representando $77,6 \%$ da população elegível.

A coleta de dados ocorreu no período de maio a junho de 2015. Utilizou-se um instrumento para o levantamento das características biopsicossociais e do trabalho dos participantes do estudo, composto por questões relacionadas ao sexo, idade, estado civil, possuir filhos, formação, tempo de formado, tempo de trabalho na instituição, tempo de trabalho na atual unidade, turno de trabalho, vínculo com mais de um emprego, motivo da atuação na unidade, intenção de deixar o emprego no hospital, intenção de deixar a enfermagem e participação em algum erro na assistência, com e sem danos, nas últimas quatro semanas.

Para o levantamento do estresse utilizou-se a Escala de Estresse no Trabalho (ETT), validada por Tamayo e Paschoa/ ${ }^{8}$, elaborada a partir da análise da literatura sobre estressores organizacionais de natureza psicossocial e sobre reações psicológicas ao estresse, com diferentes trabalhadores de empresas públicas e privadas. A EET possui 23 itens que apresentam, no mesmo item e ao mesmo tempo, um estressor e uma reação. Para cada item existe uma escala de cinco pontos, variando desde "discordo totalmente" a "concordo totalmente".

O nível de estresse de cada participante é obtido pela soma total dos itens pontuados na escala; assim, quanto maior o escore, maior o nível de estresse. A pontuação varia de um mínimo de 23 até um máximo de 115 pontos. Para a análise, realizou-se a estratificação pelo quartil ${ }^{9}$. Para essas análises, os níveis de estresse foram dicotomizados pela média da pontuação em alto e baixo ${ }^{6}$. Neste estudo, os valores dos interquartis estratificados foram: pontuações $\leq 36,25$ = baixo estresse; $\geq 36,26$ e $\leq 60,74$ = médio estresse; e, $\leq 60,75$ = alto estresse.

As informações coletadas para o estudo foram digitadas, por dois digitadores indepentendes em planilhas Excel, com posterior checagem das inconsistências. Posteriormente, os dados foram processados pelo Statistical Package for the Social Sciences (SPSS), versão 18.0, com validação e checagem da consistência dos dados.

As características biopsicossociais e do trabalho da equipe de enfermagem foram apresentadas com frequências absolutas (n), relativas (\%), mínimo, máximo e mediana. A confiabilidade do instrumento de estresse foi analisada com o 
Alfa de Cronbach. Considerou-se inadequados valores de alfa inferiores a 0,60; adequados com algumas carências os coeficientes entre 0,60 e 0,70; adequados entre 0,70 e 0,80; bons entre 0,80 e 0,85; e, excelentes os superiores a 0,8510. Para analisar a distribuição dos dados, utilizou-se o teste de Kolmogorov-Smirnov.

Para a identificação da associação entre níveis de estresse e variáveis qualitativas biopsicossociais e do trabalho da equipe de enfermagem foi utilizado o teste do Qui-Quadrado. O nível de significância adotado para todos os testes foi de $5 \%$.

Este estudo seguiu as diretrizes em observância à legislação sobre pesquisas envolvendo seres humanos, sendo autorizado e aprovado pelo Comitê de Ética em Pesquisa da instituição estudada, sob parecer número 1.035.521 e CAAE: 43176215.3 .0000 .5346$.

\section{RESULTADOS}

Participaram do estudo 52 profissionais da equipe de enfermagem da UCC, sendo 15 enfermeiros (28,8\%), 32 técnicos de enfermagem (61,6\%) e 5 auxiliares de enfermagem (9,6\%). A mediana da idade dos profissionais foi 39,5 anos, com mínimo de 25 e máximo de 57 anos.

Quanto às características biopsicossociais, $45(86,5 \%)$ são do sexo feminino, $37(71,2 \%)$ possuem companheiro e $37(71,2 \%)$ têm filhos. Com relação a formação, predominou curso técnico ( $n=22 ; 42,2 \%$ ) e de aprimoramento/ especialização ( $n=13 ; 25,0 \%)$, seguidos de graduação $(n=11 ; 21,2 \%)$, mestrado $(n=4 ; 7,7 \%)$ e auxiliar de enfermagem $(n=2 ; 3,8 \%)$. No que se refere ao perfil laboral, a maior parte dos profissionais atua no turno da manhã ( $n=21 ; 40,40 \%)$, com horário fixo ( $n=28 ; 53,85 \%)$ e sem outro vínculo empregatício ( $n=47 ; 90,40 \%)$.

A Tabela 1 apresenta a distribuição da equipe de enfermagem da UCC segundo a escolha pelo trabalho, percepção motivacional do trabalho, da instituição e da profissão.

Tabela 1 - Distribuição da equipe de enfermagem segundo a escolha pelo trabalho, percepção motivacional do trabalho, da instituição e da profissão. Santa Maria, RS, 2015.

\begin{tabular}{lll}
\hline Variáveis & $\mathbf{n}$ & $\%$ \\
\hline Motivo para trabalhar na clínica cirúrgica & & \\
Escolheu & 11 & 21,2 \\
Não havia vaga em outro setor & 10 & 19,2 \\
Remanejo & 19 & 36,5 \\
Outro & 12 & 23,1 \\
Sente-se disposto para trabalhar & & 88,5 \\
Sim & 46 & 11,5 \\
Não & 06 &
\end{tabular}


Gosta de trabalhar na clínica cirúrgica

Sim

Não

02

3,8

Não respondeu

01

1,9

Sente-se satisfeito em trabalhar na clínica cirúrgica

Sim

49

94,2

Não

03

5,8

Possui intenção de deixar a instituição

Sim

06

11,5

Não

46

88,5

Possui intenção de deixar a enfermagem

Sim

06

11,5

Não

46

88,5

Evidencia-se que o principal motivo de os profissionais da equipe de enfermagem estarem trabalhando na UCC é o remanejo ( $n=19 ; 36,5 \%)$. Também, $88,5 \%(n=46)$ da equipe sente-se disposta a trabalhar, $94,2 \%(n=49)$ gosta de trabalhar na clínica cirúrgica e 94,2\% (n=49) estão satisfeitos em trabalhar no setor e não pretendem deixar a instituição $(n=46 ; 88,5 \%)$, nem a enfermagem $(n=46 ; 88,5 \%)$.

Em relação ao tempo de formado, a mediana é de 12,5 anos, a mediana do tempo de trabalho na instituição é de 0,7 e a mediana do tempo de atuação na UCC é de 2,6 anos.

No que concerne a cometer algum erro com dano ou lesão ao paciente, nas últimas quatro semanas, ninguém da equipe respondeu afirmativamente à questão. Contudo, $5,8 \%(n=3)$ respondeu ter cometido erro na assistência, mas que este foi percebido e não acarretou em prejuízo ou dano ao paciente.

Quanto aos resultados acerca do estresse, a confiabilidade da EET foi de 0,925. No que se refere ao nível de estresse, dos 52 profissionais da equipe de enfermagem da UCC, $50 \%$ (26) apresentaram médio nível de estresse, $25 \%$ (13) estavam com baixo nível e 25\% (13) apresentaram alto nível de estresse. A Tabela 2 apresenta as questões mais e menos pontuadas da EET.

Tabela 2 - Estatísticas descritivas das questões mais e menos pontuadas de estresse (EET) pela equipe de enfermagem. Santa Maria, RS, 2015.

\section{Questões do instrumento EET}

Soma Média Desvio

Padrão

\section{Questões mais pontuadas}

Q22 - "O tempo insuficiente para realizar meu volume de trabalho deixa-me nervoso" 142

Q01 - "A forma como as tarefas são atribuídas em minha área tem me deixado nervoso" 138

$2,7 \quad 1,2$

Q12 - "Fico irritado com a discriminação/favoritismo no meu ambiente de trabalho"

136

$2,6 \quad 1,2$

$2,6 \quad 1,2$




\section{Questões menos pontuadas}

Soma Média Desvio

Padrão

Q17 - "Tenho me sentido incomodado por trabalhar em tarefas abaixo do meu nível de habilidade"

Q20 - "Tenho estado nervoso por meu superior me dar ordens contraditórias"

Q23 - "Fico incomodado por meu superior evitar me incumbir de responsabilidades importantes"
$84 \quad 1,6 \quad 0,8$

$86 \quad 1,6 \quad 0,8$

$91 \quad 1,7 \quad 0,9$

A análise da associação entre o estresse medido pelo instrumento EET (imputado pelas médias em alto e baixo) com as variáveis sexo, estado civil, número de filhos, categoria profissional, maior formação, horário fixo de trabalho e outro vínculo empregatício será apresentada na Tabela 3.

Tabela 3 - Associação entre estresse e variáveis biopsicossociais e do trabalho da equipe de enfermagem. Santa Maria, RS, 2015.

\begin{tabular}{|c|c|c|c|c|}
\hline \multirow[t]{2}{*}{ Variáveis } & & \multicolumn{2}{|c|}{$\begin{array}{ll}n & (\%)\end{array}$} & \multirow[t]{2}{*}{$p^{*}$} \\
\hline & & Baixo Estresse & Alto Estresse & \\
\hline \multirow[t]{2}{*}{ Sexo } & Masculino & $2(7,1)$ & $5(20,8)$ & 0,149 \\
\hline & Feminino & $26(92,9)$ & $19(79,2)$ & \\
\hline \multirow[t]{2}{*}{ Estado civil } & Sem companheiro (a) & $5(17,9)$ & $10(41,7)$ & 0,059 \\
\hline & Com companheiro (a) & $23(82,1)$ & $14(58,3)$ & \\
\hline \multirow[t]{2}{*}{ Possuir filhos } & Não & $6(21,4)$ & $9(37,5)$ & 0,202 \\
\hline & Sim & $22(78,6)$ & $15(62,5)$ & \\
\hline Categoria & Enfermeiro & $6(21,4)$ & $9(37,5)$ & 0,285 \\
\hline \multirow[t]{2}{*}{ profissional } & Téc. em enfermagem & $20(71,4)$ & $12(50,0)$ & \\
\hline & Aux. de enfermagem & $2(7,1)$ & $3(12,5)$ & \\
\hline \multirow[t]{4}{*}{ Maior formação } & Curso técnico & $13(46,4)$ & $9(37,5)$ & 0,462 \\
\hline & Graduação & $5(17,9)$ & $6(25,0)$ & \\
\hline & Especialização & $7(25,0)$ & $6(25,0)$ & \\
\hline & Mestrado & $1(3,6)$ & $3(12,5)$ & \\
\hline \multirow{5}{*}{$\begin{array}{l}\text { Formação } \\
\text { em andamento }\end{array}$} & Curso técnico & $0(0,0)$ & $1(4,2)$ & 0,404 \\
\hline & Graduação & $3(10,7)$ & $4(16,7)$ & \\
\hline & Especialização & $3(10,7)$ & $0(0,0)$ & \\
\hline & Mestrado & $2(7,1)$ & $1(4,2)$ & \\
\hline & Doutorado & $0(0,0)$ & $1(4,2)$ & \\
\hline \multirow[t]{3}{*}{ Turno } & Manhã & $12(42,9)$ & $9(37,5)$ & 0,916 \\
\hline & Tarde & $6(21,4)$ & $6(25,0)$ & \\
\hline & Noite & $10(35,7)$ & $9(37,5)$ & \\
\hline \multirow[t]{2}{*}{ Possuir horário fixo } & Sim & $16(57,1)$ & $12(50,0)$ & 0,518 \\
\hline & Não & $11(39,3)$ & $12(50,0)$ & \\
\hline \multirow{4}{*}{$\begin{array}{l}\text { Possuir outro } \\
\text { vínculo empregatício } \\
\text { Tipo de vínculo }\end{array}$} & Sim & $3(10,7)$ & $2(8,3)$ & 0,772 \\
\hline & Não & $25(89,3)$ & $22(91,7)$ & \\
\hline & CLT & $17(60,7)$ & $14(58,3)$ & 0,862 \\
\hline & Estatutário & $11(39,3)$ & $10(41,7)$ & \\
\hline \multirow{2}{*}{$\begin{array}{l}\text { Satisfação } \\
\text { no trabalho }\end{array}$} & Sim & $28(100)$ & $21(87,5)$ & 0,054 \\
\hline & Não & $0(0,0)$ & $3(12,5)$ & \\
\hline
\end{tabular}

p*Teste Qui-quadrado. 
Verifica-se que não houve associação estatisticamente significativa entre nível de estresse medido e as variáveis biopsicossociais e do trabalho dos profissionais de enfermagem da UCC.

\section{DISCUSSÃO}

A partir da análise das características biopsicossociais da equipe de enfermagem da UCC, comprovou-se, quanto ao sexo, a predominância de mulheres. A predominância de mulheres na enfermagem já é característica da profissão, uma vez que ainda está arraigado a um pensamento antigo, quando a enfermagem era praticada somente para as mulheres, atrelado ao cuidado ainda da época de Florence Nigthigale, precursora da profissão. Vale ressaltar que a presença do sexo masculino na profissão de enfermagem tem crescido nos últimos anos. Os achados desta pesquisa corroboram outros estudos, com a predominância do sexo feminino atingindo percentuais de $81,3 \%$ e $87,5 \%$.

No que se refere ao estado civil e número de filhos, o estudo apontou predominância de profissionais que possuem companheiros (as) e filhos. Dados que, quando comparados, ultrapassam os percentuais de pesquisa ${ }^{13}$ que evidenciou 50,5\% para profissionais com companheiro e 63,1\% com filhos. Não obstante, vai ao encontro dos resultados de outro estudo ${ }^{14}$, em que $72,4 \%$ da equipe possuíam companheiro e $65,5 \%$ com filhos. Nesta perspectiva, os filhos e a família podem servir de suporte para os profissionais e, com isso, representar auxílio para melhor enfrentamento dos estressores laborais.

Abrangendo a formação prevalente, a predominância foi de curso técnico. Dados apontam que vários dos profissionais atuantes em determinada área possuem formação maior, porém, trabalham em níveis e categorias diferentes em virtude de seu atual cargo ser de concurso público, o que lhes dá mais estabilidade ${ }^{14}$. Corroborando, a procura por graduação, especialização e pós-graduação na área de enfermagem vem aumentando no Brasil, resultado do crescimento do número de cursos e programas com este enfoque. Vale ressaltar, ainda, que há uma preocupação por parte dos avaliadores destes programas em manter suas qualidades e excelência ${ }^{15}$.

Por conseguinte, o turno predominante da atuação da equipe de enfermagem foi o da manhã, sendo que maior parte dos profissionais possuem horário fixo e não possuem outro vínculo empregatício. Percebe-se uma distribuição adequada dos profissionais contemplando seus turnos. Ainda, o horário fixo contribui para uma melhor organização e logística da unidade. Em contrapartida, alguns profissionais não encerram sua jornada de trabalho ao final de um plantão, uma vez que têm trabalho doméstico e atenção e cuidados aos filhos ${ }^{16}$.

Neste estudo, percebeu-se que a maioria dos participantes não possui outro vínculo empregatício, o que se contrapõe a outros achados, onde o percentual de profissionais com outro vínculo empregatício chega a 21,9\% e 53,4\% $\%^{11,14}$. A dupla jornada de trabalho é uma das opções escolhidas pelos profissionais na busca por uma melhor remuneração e 
qualidade de vida; entretanto, considera-se que esta acarreta em sobrecarga de trabalho e, consequentemente, influencia na qualidade dos cuidados prestados ${ }^{17}$.

Na distribuição da equipe de enfermagem segundo a percepção motivacional do trabalho, da instituição e da profissão surge o remanejo como sendo o principal motivo dos profissionais da equipe de enfermagem estarem atuando na unidade. Percebe-se que este motivo não influencia na satisfação e disposição em atuar no setor, já que a maioria da equipe se sente disposta a trabalhar, gosta de trabalhar na UCC e não pretende deixar a instituição, tão pouco a enfermagem.

Abrangendo o estresse e a satisfação profissional, por um lado, a condição favorável de estar satisfeito onde está atuando representa aspecto positivo, pois a pessoa enfrenta melhor o estresse em determinado ambiente. Em contraponto, o fato de poder escolher onde trabalhar, o que não se identificou no presente estudo, pode ser um estressor, cabendo ao indivíduo enfrentar a não opção pelo trabalho como um estressor ou como um evento positivo ${ }^{13}$.

A mediana, em anos, do tempo de formação da equipe de enfermagem da clínica cirúrgica foi de 12,5, o que se mostra superior a outros achados onde essa média é de 11,6 anos de formação, podendo esta experiência profissional ser considerada destaque ${ }^{13}$. Também, a mediana do tempo de trabalho na instituição foi de 0,7 e a mediana do tempo de atuação em clínica cirúrgica de 2,6 anos; para estes últimos dados, no cenário do estudo, não se encontram estudos brasileiros disponíveis para possíveis comparações a respeito. No entanto, observa-se que a experiência de trabalho pode ser positiva no que tange a avaliação e enfrentamento dos estressores no trabalho ${ }^{13}$.

Contemplando o cometer algum erro, os participantes negam ter cometido algum erro com dano ou lesão ao paciente nas últimas quatro semanas. Porém, 5,8\% alegam ter cometido erros que não causaram danos aos pacientes, percentual superior a outro estudo ${ }^{13}$. Cabe a reflexão acerca do cometer ou não o erro e este ser informado e notificado, visto que ainda é presente o pensamento de cultura punitiva nos serviços de saúde, o que leva à insegurança por parte dos profissionais em se expor. É valido ressaltar que, independentemente de o erro ser ou não com dano/lesão ao paciente, o mesmo deve ser notificado o quanto antes. Com isso, visa-se verificar falhas no sistema para que posteriormente essas sejam corrigidas e para evitar que aconteçam novos erros na assistência em saúde ${ }^{13,18}$.

Soma-se a isso que a quantidade de notificações encontradas é bastante reduzida quando comparada aos erros cometidos e identificados em estudos ${ }^{18}$. Considerando que a cultura punitiva interfere fortemente nesses ocorridos, 0 apoio da instituição pode ser visto como grande aliado na busca pela redução dessa cultura punitiva. Em contraponto, muitos profissionais ainda desconhecem maneiras de notificar os erros cometidos ${ }^{18}$.

Os resultados encontrados com a equipe de enfermagem da clínica cirúrgica deste estudo podem ser aproximados dos fatores associados ao estresse pelos estudos citados quando se analisam as questões mais pontuadas como mais estressantes. Nesta amostra, foram as questões relacionadas ao tempo insuficiente para realizar o volume de tarefas e a forma como as tarefas são atribuídas na área às questões mais pontuadas, o que envolve carga de trabalho e 
complexidade do setor de atuação, bem como o fato da equipe ficar irritada com a discriminação/favoritismo no seu ambiente de trabalho, abrangendo a desvalorização profissional ${ }^{13}$.

Reforçando, outros estudos apontam que os estressores para a equipe de enfermagem podem contribuir para a ocorrência de eventos adversos/incidentes ${ }^{6,17}$. Estudo que visou identificar a influência da sobrecarga de trabalho da enfermagem na ocorrência de incidentes sem lesão e eventos adversos evidenciou que $78 \%$ dos EA/l foram relacionados à esfera enfermagem, sobretudo à sobrecarga de trabalho ${ }^{17}$.

No que se refere às questões menos pontuadas, estão o incômodo por trabalhar em tarefas abaixo do nível de habilidades que o profissional possui, o nervosismo por receber ordens contraditórias de seu superior e o incômodo por seu superior não incumbir o profissional de responsabilidades importantes. Assim, de maneira geral, percebe-se que a equipe de enfermagem da UCC se preocupa em realizar todas as suas demandas assistenciais e pouco considera o fato de serem contrariados por supervisores ou por trabalharem em demandas que não lhes competem.

Evidenciou-se que não houve associação significativa estatisticamente entre nível de estresse e as variáveis biopsicossociais e do trabalho dos profissionais de enfermagem, conforme o teste Qui-quadrado. Embora não se tenha constatado tais associações, alguns estudos indicam que as mulheres estão mais expostas e suscetíveis ao estresse, já que possuem, em sua grande maioria, outros papéis sociais, tais como: serem mães e/ou esposas, o que as faz ainda levarem todas essas preocupações para o trabalho, e vice-versa. Outro estudo aponta que, além dos papéis sociais que a mulher exerce, algumas mantêm a busca pela qualificação profissional, contribuindo assim para o aumento dos estressores ${ }^{13,19}$.

Estudo indica que ter companheiro e filhos ${ }^{13}$ pode representar fatores de proteção, indicando apoio, segurança e, consequentemente, estímulo para enfrentar o estresse. É possível que as preocupações com educação e segurança dos filhos acabem interferindo na avaliação dos estressores no ambiente de trabalho.

No que concerne à categoria profissional e formação, embora não estatisticamente significativas, percebeu-se predominância de trabalhadores de nível técnico com estresse mais baixo, o que pode ser justificado pelas atribuições e responsabilidades de cada categoria profissional. Por outro lado, estudos consideram a pós-graduação como estratégia para auxiliar no enfrentamento do estresse, bem como estimular os profissionais a possuírem um maior senso crítico e um olhar ampliado para as práticas laborais, aplicando, então, tais práticas para o bem de si mesmo e da sociedade ${ }^{13,19}$.

Por conseguinte, se há formação em andamento, maior é o nível de estresse do profissional, o que pode ser justificado pelo desempenho do papel de estudante por parte desses profissionais e as preocupações em atender as demandas dos cursos de formação. Além disso, a variável horário fixo também não teve associação com o estresse, embora possa ser outro fator de preocupação entre os profissionais estudantes, uma vez que lhes é exigido conciliar horários de estudo e trabalho. 
A variável vínculo empregatício não demonstrou diferença estatística significativa entre os indivíduos que possuem ou não outro emprego, porém, no estudo, verificou-se que a maioria dos sujeitos está satisfeita em seu trabalho e gosta de atuar no setor e profissão. A escolha por manter outro emprego agrega a representatividade de ter um acréscimo salarial mensal, fato frequente na enfermagem frente à desvalorização da categoria, que é uma realidade ${ }^{13}$.

Posto isso, este estudo avança no que tange ao conhecimento produzido relacionado à equipe de enfermagem que atua em UCC, ao passo que evidencia fatores que podem estar associados ao estresse dos profissionais. $\mathrm{O}$ ambiente estudado apresenta características que podem ser estressantes. Nesta perspectiva, torna-se necessário que novas pesquisas sejam desenvolvidas com vistas a identificar estratégias de enfrentamento para o estresse de profissionais que atuam em cenário cirúrgico.

\section{CONSIDERAÇÕES FINAIS}

O estresse prevalente na equipe de enfermagem da UCC foi o de nível médio, sendo que apenas um terço da equipe estava com nível baixo de estresse, ou seja, percebeu-se que esta unidade se caracteriza por ser estressante aos profissionais, ainda que não foram evidenciadas associações estatisticamente significativas. Apesar disso, verificou-se que o estresse é presente no cenário pesquisado, o que indica a necessidade de novas pesquisas que visem propor estratégias interventivas com vistas a melhorar a qualidade de vida no trabalho, favorecendo o enfrentamento dos estressores.

Por fim, considera-se que algumas limitações podem ter dificultado a identificação de associação entre estresse e as características biopsicossociais e do trabalho da equipe de enfermagem do estudo, como o tamanho da amostra. Ressalta-se que na literatura brasileira são escassos os estudos que avaliam o estresse da equipe de enfermagem de cenário cirúrgico, especificamente, o que dificultou a discussão dos dados no presente estudo. Ainda, não foi possível evidenciar a relação de causa e efeito entre exposição e desfecho, limitação relacionada aos estudos transversais.

\section{REFERÊNCIAS}

1. Andrade RVS, Costa ORS. Estresse ocupacional em profissionais de saúde: um estudo com a equipe de enfermagem da unidade de terapia intensiva - UTI. Rev. Ciências em Saúde, 4 (4):11, 2014.

2. Nodari NL, Flor SRA, Ribeiro AS, Carvalho GJR. Estresse, conceitos, manifestações e avaliação em saúde: revisão de literatura. Revista Saúde e Desenvolvimento Humano, 2(1): 61-74, 2014. 
3. Silva EFL, Moura MLC. Estresse nas relações enfermeiro/paciente: revisão integrativa. Rev enferm UFPE on line, 8(7):2140-8, jul., 2014.

4. Puerto CJ, Soler ML, Montesinos LJM, Marcos PA, Chorda GMV. Uma nova contribuição para a classificação dos fatores estresores que afetam os profissionais de enfermagem. Revista Latino - Americana de Enfermagem, 25 (2895): $1-7,2017$.

5. Paixão GPN, Evangelista JT, Oliveira AK, Salgado AM, Bittencourt SI. Estresse da equipe de enfermagem e sua influência na produtividade. C\&D-Revista Eletrônica da Fainor, 6 (1): 79-92, 2013.

6. Andolhe R, Barbosa LR, Oliveira ME, Costa SLA, Padilha GK. Estresse, coping e burnout da Equipe de Enfermagem de Unidades de Terapia Intensiva: fatores associados. Rev Esc Enferm USP, 49(Esp): 58-64, 2015.

7. Mello RCC, Reis LB, Ramos FP. Estresse em Profissionais de Enfermagem: Importância da Variável Clima Organizacional. Revista Interinstitucional de Psicologia, 11(2):193-207, 2018.

8. Paschoal T, Tamayo, A. Validação da Escala de Estresse no trabalho. Estud Psicol, 9 (1): 45-52, 2004.

9. Trigo TR. Validade fatorial do Maslach Burnout Inventory-Human Services Survey (MBI-HSS) em uma amostra brasileira de auxiliares de enfermagem de um hospital universitário: influência da depressão [dissertação]. São Paulo: Universidade de São Paulo, Faculdade de Medicina; 2011.

10. Prieto G, Muñiz, J. Un modelo para evaluar la calidad de los testsutilizados en España. Papeles del Psicólogo, (77):65-72, 2000

11. Paglione NL, Vannuchi MTO, Tenani MNF, Pissinati CSP. Satisfação profissional da equipe de enfermagem do centro cirúrgico em hospital público de média complexidade. Rev enferm UFPE on line, 10(3):1080-7, 2016.

12. Rolim CSS. Estresse e síndrome de burnout em profissionais de enfermagem. Rev. Bras. Pesq. Saúde, $15(3):$ 103-113, 2013. 
13. Andolhe R. Segurança do paciente em unidades de terapia intensiva: estresse, coping e burnout da equipe de enfermagem e ocorrência de eventos adversos e incidentes [tese]. São Paulo: Escola de Enfermagem; 2013.

14. Silva ROC, Batista KM, Grazziano ES. Personalidade resistente nas equipes médica e de enfermagem em centro cirúrgico. Rev. SOBECC, 19 (4): 214-218, 2014.

15. Scochi CGS, Munari DB, Gelbcke FL, Erdmann AL, Gutiérrez MGR, Rodrigues RAP. Pós-Graduação Stricto Sensu em Enfermagem no Brasil: avanços e perspectivas. Rev Bras Enferm, v. 66 (esp), p. 80-9, 2013.

16. Oliveira GM, Silva RM, Moraes Filho IM, Guido LA. Influência do turno de trabalho na qualidade de vida dos profissionais de enfermagem de um hospital público do noroeste do Mato Grosso- MT. Rev. Cient. Sena Aires, 5(1): 4-20, 2016.

17. Novaretti MCZ, Santos EV, Quitério LM, Daud-Gallotti RM. Sobrecarga de trabalho da Enfermagem e incidentes e eventos adversos em pacientes internados em UTI. Rev. Bras Enferm, 67(5): 692-9., 2014.

18. Souza VS, Kawamoto AM, Oliveira JLC, Tonini NS, Fernandes LM, Nicola AL. Erros e eventos adversos: a interface com a cultura de segurança dos profissionais de saúde. Cogitare Enferm, 20(3): 475-482, 2015.

19. Rodrigues CCFM, Salvador PTCO, Assis YMS, Gomes ATL, Bezerril MS, Santos VEP. Estresse entre os membros da equipe de enfermagem. Rev enferm UFPE on line, 11(2): 601-8, 2017. 